\title{
Coronavirus - Drug Discovery and Therapeutic Drug Monitoring Options
}

Kashif Aziz Ahmad ${ }^{1 *}$, Saleha Akram Nizami ${ }^{2}$, Muhammad Haroon Ghous ${ }^{3}$

${ }^{1}$ Assistant professor of Medicine, Unit-3, Lahore General Hospital / PGMI / AMC. Pakistan.

${ }^{2}$ University of Lahore, Pakistan.

${ }^{3}$ Associate Professor \& Head of Department of Urology, University College of Medicine, University of Lahore

*Corresponding Author: Kashif Aziz Ahmad, Assistant professor of Medicine, Unit-3, Lahore General Hospital / PGMI / AMC. Pakistan. Received date: November 09, 2021; Accepted date: December 13, 2021; Published date: January 05, 2022

Citation: Kashif Aziz Ahmad, Saleha Akram Nizami, Muhammad Haroon Ghous (2022) Coronavirus - Drug Discovery and Therapeutic Drug Monitoring Options. J. Pharmaceutics and Pharmacology Research, 5(2); DOI: 10.31579/2693-7247/044

Copyright: () 2022 Kashif Aziz Ahmad, This is an open access article distributed under the Creative Commons Attribution License, which permits unrestricted use, distribution, and reproduction in any medium, provided the original work is properly cited.

\begin{abstract}
Summary
COVID-19 is basically a medium size RNA virus and the nucleic acid is about $30 \mathrm{~kb}$ long, positive in sense, single stranded and polyadenylated. The RNA which is found in this virus is the largest known RNA and codes for a large polyprotein. In addition, coronaviruses are capable of genetic recombination if 2 viruses infect the same cell at the same time. SARS-CoV emerged first in southern China and rapidly spread around the globe in 2002-2003. In November 2002, an unusual epidemic of atypical pneumonia with a high rate of nosocomial transmission to healthcare workers occurred in Foshan, Guangdong, China. In March 2003, a novel CoV was confirmed to be the causative agent for SARS, and was thus named SARS-CoV. Despite the report of a large number of virus-based and host-based treatment options with potent in vitro activities for SARS and MERS, only a few are likely to fulfil their potential in the clinical setting in the foreseeable future. Most drugs have one or more major limitations that prevent them from proceeding beyond the in vitro stage. First, many drugs have high EC50/Cmax ratios at clinically relevant dosages
\end{abstract}

Key words: drug discovery; therapeutic drug; COVID-19

\section{Introduction}

The history of corona virus family is very old, it begins in 1965 when Tyrrell and Bynoe found that there was a virus family who damage the respiratory pathway. This virus was named as B814 in that time. It was transmitted from animals to humans. Now, in 2020 there is a virus COVID-19 which is also belongs to the family of corona virus is infected the whole world. People all around the world facing the situation of pandemic. This virus effected the whole world and do not believe in racism.

COVID-19 is basically a medium size RNA virus and the nucleic acid is about $30 \mathrm{~kb}$ long, positive in sense, single stranded and polyadenylated. The RNA which is found in this virus is the largest known RNA and codes for a large polyprotein. In addition, coronaviruses are capable of genetic recombination if 2 viruses infect the same cell at the same time.

The most common symptoms of COVID-19 is cold, flu, fever and infection in lungs. There are different stages in the attacking of this virus. At stage one and at the start patient just feel flu and temperature just like common cold and flu. But after seven days it becomes more worse and patient feels shortness of breath and dry cough. At advanced stage the patients become also suffered from pneumonia. There is no vaccine and antiviral therapy until now.
In this short communication we will talk about current situation of COVID-19 in Pakistan. It was basically starts from China from December 2019, when there was a person who died in Wuhan (a city of China) due to an unknown virus. What started as an epidemic mainly limited to China has now become a truly global pandemic. There have now been over 392,331 confirmed cases and 17,156 deaths, according the John Hopkins University Covid-19 dashboard, which collates information from national and international health authorities. The disease has been detected in more 196 countries and territories, with Italy, the US and Spain experiencing the most widespread outbreaks outside of China. There were 438,441 cases from which 19,650 died and 111,877 were recovered all around the world.

\section{Epidemiology}

SARS-CoV started first in southern China and quickly spread the world over in 2002-2003. In November 2002, an irregular pandemic of atypical pneumonia with a high pace of nosocomial transmission to human services laborers happened in Foshan, Guangdong, China. In March 2003, a novel $\mathrm{CoV}$ was affirmed to be the causative specialist for SARS, and was in this manner named SARS-CoV. The disengagement of a novel $\beta \mathrm{CoV}$ from a patient in Jeddah, Saudi Arabia, who passed on of extreme pneumonia and multi-organ disappointment in June 2012, was first detailed in September 2012. At first named 'human coronavirus Erasmus Medical Center', the infection was later renamed MERS-CoV by global accord, and the sickness was called Middle East respiratory syn-drome 
(MERS). Review examination of a group of nosocomial cases in April 2012 in Jordan affirmed that MERS-CoV was likewise liable for that flare-up. In the course of recent years, MERS-CoV has kept on spreading inside and past the Middle East, and there are progressing reports of inconsistent cases and network and social insurance related groups of tainted people in the Middle East, particularly in Saudi Arabia and the United Arab Emirate. Travel-related cases and groups have likewise been progressively given an account of different mainlands. Starting at 9 October 2015, 1,593 lab affirmed instances of MERS, including 568 passings, have been accounted for to the World Health Organization.

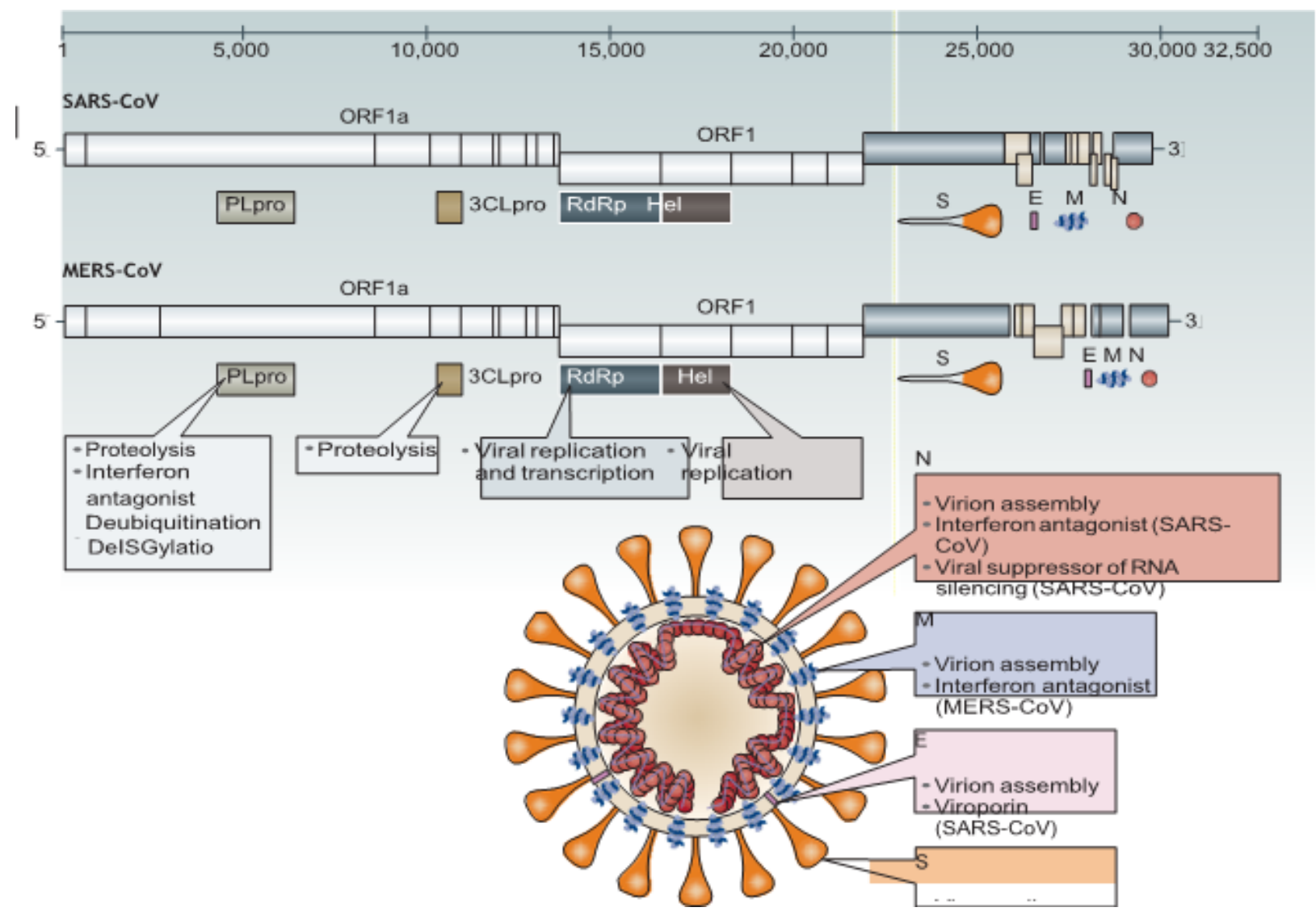

\section{Non-structural proteins Structural and accessory proteins}

Figure 1: Genomes and structures of SARS-CoV and MERS-CoV.

The typical coronavirus ( $\mathrm{CoV}$ ) genome is a single-stranded, non-segmented RNA genome, which is approximately $26-32 \mathrm{~kb}$. It contains $5^{\prime}$-methylated caps and $3^{\prime}$-polyadenylated tails and is arranged in the order of $5^{\prime}$, replicase genes, genes encoding structural proteins (spike glycoprotein $(S)$, envelope protein $(E)$, membrane protein $(\mathrm{M})$ and nucleocapsid protein $(\mathrm{N})$ ), poly adenylated tail and then the $3^{\prime}$ end. The partially overlapping 5'-terminal open reading frame $1 \mathrm{a} / \mathrm{b}(\mathrm{ORF} 1 \mathrm{a} / \mathrm{b})$ is within the $5^{\prime}$ two-thirds of the CoV genomeandencodesthelargereplicase plyprotein $1 \mathrm{a}(\mathrm{pp} 1 \mathrm{a})$ andpp $1 \mathrm{ab}$.

\section{Approaches to anti-CoV drug screening}

The main two human-pathogenic CoVs known before the SARS pestilence were $\mathrm{HCoV}-229 \mathrm{E}$ and $\mathrm{HCoV}-\mathrm{OC} 43$, which as a rule because self-constraining upper respiratory tract contaminations. Along these lines, analysts and examination offices, particularly those associated with antiviral turn of events, were underprepared when SARS-CoV out of nowhere emerged in 2003. In this manner, three general methodologies were utilized to find potential enemy of $\mathrm{CoV}$ treatment alternatives for human-pathogenic CoVs particularly SARS-CoV and the rising MERS-CoV.
The main way to deal with sedate revelation is to test existing wide range antiviral medications that have been utilized to treat other viral diseases by utilizing standard examines that measure the impacts of these medications on the cytopathi city, infection yield and plaque development of live and additionally pseudo composed CoVs. Instances of medications distinguished utilizing this methodology incorporate interferon alfa, interferon beta, interferon gamma, ribavirin and inhibitors of cyclophilin. These medications have the undeniable preferred position of being promptly accessible with known pharmacokinetic and pharmacodynamic properties, symptoms and dosing regimens. Nonetheless, they don't have explicit enemy of $\mathrm{CoV}$ impacts and might be related with serious antagonistic impacts. The second enemy of $\mathrm{CoV}$ medicate revelation approach includes the screening of substance libraries involving huge quantities of existing mixes or databases that contain data on transcriptional marks in various cell lines. This methodology gives quick, high-throughput screening of numerous promptly accessible aggravates that would then be able to be additionally assessed by hostile to viral tests. Different classes of medications have been distinguished in these medication repurposing programs, including numerous that have significant physiological as well as immunological impacts, for example, those that influence synapse guideline, the estrogen receptor, 
kinase flagging, lipid or sterol digestion, protein handling and DNA amalgamation or fix.

\section{Virus-based anti-CoV treatment options}

Nucleosides and nucleotides are the building blocks of viral nucleic acids. Medications that target nucleosides or nucleotides as well as viral nucleic acids by and large have expansive range movement against a wide scope of CoVs and different viruses. Mycophenolate mofetil is an enemy of dismissal tranquilize that represses inosine monophos-phate dehydrogenase and the union of guanine monophosphate. The dynamic compound, mycophenolic corrosive, displays antiviral action in vitro against different viruses, including hepatitis B infection (HBV), hepatitis C infection (HCV) and arbo viruses. Mycophenolic corrosive was distinguished as a potential enemy of MERS-CoV sedate utilizing high-throughput screening and has powerful enemy of MERS-CoV action in vitro122. In any case, an ensuing report in a non-human primate model demonstrated that MERS-CoV-tainted normal marmosets rewarded with mycophenolate mofetil had a more regrettable result with progressively extreme illness and higher viral loads in necropsied lung and extra pneumonic tissues than untreated creatures. Renal transplant beneficiaries who were on upkeep mycophenolate mofetil treatment additionally created serious or deadly MERS. Subsequently, the standard measurement of mycophenolate mofetil monotherapy is probably not going to be helpful for prophylaxis or treatment of $\mathrm{CoV}$ contaminations.

\section{Advancement of MERS-CoV immunizations}

Quick diagnostics and compelling immunizations are regularly correlative to antiviral treatment in the control of pandemics brought about by developing viruses. In spite of the fact that there has not been any new human SARS case for more than 10 years, inconsistent cases and groups of MERS keep on happening in the Middle East inferable from the diligence of zoonotic sources in endemic territories, and these cases spread to different areas. Powerful MERS-CoV antibodies are basic for intruding on the chain of transmission from creature stores and contaminated people to helpless hosts. Live-constricted antibodies, which have been recently assessed in creature models for SARS, may be related with dispersed disease in resistant traded off patients. Inactivated antibodies may be related with immunopathology during creature challenge. These are un great methodologies for MERS antibody advancement on the grounds that a considerable extent of patients with serious MERS have comorbidities or fundamental resistant bargaining conditions. Other immunization systems for MERS that utilization DNA plasmids, viral vectors, nanoparticles, infection like particles and recombinant protein subunits are being developed and some have been assessed in creature models. The accessibility of a sheltered and powerful MERS-CoV antibody for dromedary camels and non-invulnerable people at high danger of camel contact in endemic locales, for example, the Middle East and the more prominent Horn of Africa would be a significant measure for controlling the progressing pandemic.

Reasonable creature models are particularly significant for testing hostile to CoV drugs in light of the fact that the vast majority of these medications have not been utilized in people. As opposed to the set number of creature models set up for the mellow diseases brought about by $\mathrm{HCoV}-229 \mathrm{E}, \mathrm{HCoV}-\mathrm{OC} 43$, HCoV-NL63 and HCoVHKU1, different little creature and non-human primate models have been assessed for investigations of the pathogenesis and treatment of SARS and MERS.

\section{Conclusion}

Despite the report of a large number of virus-based and host-based treatment options with potent in vitro activities for SARS and MERS, only a few are likely to fulfil their potential in the clinical setting in the foreseeable future. Most medications have at least one significant impediments that keep them from continuing past the in vitro stage. In the first place, numerous medications have high EC50/Cmax proportions at clinically significant measurements. Instances of such medications incorporate cyclosporine, chlorpromazine and interferon alfa. Second, some have extreme symptoms or cause immunosuppression. For instance, the utilization of high-portion ribavirin might be related with haemolytic iron deficiency, neutropenia, teratogenicity and cardiorespiratory trouble. MERSCoV-contaminated basic marmosets rewarded with mycophenolate mofetil built up a deadly disease with significantly higher viral burdens in their lungs and extra aspiratory tissues than untreated controls did128. Operators focusing on have flagging pathways or receptors may instigate immunopathology. Moreover, the absence of a solid medication conveyance strategy in vivo is especially tricky for siRNAs and different operators that have not been recently utilized in people.

\section{References}

1. Guo X, et al. (2015) Systemic and mucosal immunity in mice elicited by a single immunization with human adenovirus type 5 or 41 vector-based vaccines carrying the spike protein of Middle East respiratory syndrome coronavirus. Immunology. 145: 476-484

2. Czub M, Weingartl H, Czub S, He R, Cao J. (2005) Evaluation of modified vaccinia virus Ankara based recombinant SARS vaccine in ferrets. Vaccine. 23: 2273-2279.

3. Coleman CM, et al. (2014) Purified coronavirus spike protein nanoparticles induce coronavirus neutralizing antibodies in mice. Vaccine. 32: 3169-3174.

4. Zhao G, et al. (2013) A safe and convenient pseudovirus-based inhibition assay to detect neutralizing antibodies and screen for viral entry inhibitors against the novel human coronavirus MERS-CoV. Virol. J. 10:266.

5. Du L, et al. (2013) Identification of a receptor-binding domain in the $\mathrm{S}$ protein of the novel human coronavirus Middle East respiratory syndrome coronavirus as an essential target for vaccine development. J. Virol. 87: 9939-9942.

6. Mou H, et al. (2013) The receptor binding domain of the new Middle East respiratory syndrome coronavirus maps to a 231residue region in the spike protein that efficiently elicits neutralizing antibodies. J. Virol. 87:9379-9383

7. Du L, et al. (2013) A truncated receptor-binding domain of MERS-CoV spike protein potently inhibits MERS-CoV infection and induces strong neutralizing antibody responses: implication for developing therapeutics and vaccines. PloS ONE. 8:e81587.

8. Ma C, et al. (2014) Intranasal vaccination with recombinant receptor-binding domain of MERS-CoV spike protein induces much stronger local mucosal immune responses than subcutaneous immunization: Implication for designing novel mucosal MERS vaccines. Vaccine. 32:2100-2108

9. Ma C, et al. (2014) Searching for an ideal vaccine candidate among different MERS coronavirus receptor-binding fragments-The importance of immunofocusing in subunit vaccine design. Vaccine. 32: 6170-6176.

10. Lan J, et al. (2014) Tailoring subunit vaccine immunity with adjuvant combinations and delivery routes using the Middle East respiratory coronavirus (MERS-CoV) receptor-binding domain as an antigen. PloS ONE. 9: e112602. 
11. Zhang, N. et al. (2015) Identification of an ideal adjuvant for receptor-binding domain-based subunit vaccines against Middle East respiratory syndrome coronavirus. Cell. Mol. Immunol.10.1038/cmi.2015.03.

12. Lassnig C, et al. (2005) Development of a transgenic mouse model susceptible to human coronavirus 229E. Proc. Natl Acad. Sci. USA. 102: 8275-8280.

13. Dijkman R, et al. (2009) Seroconversion to HCoV-NL63 in rhesus macaques. Viruses. 1:647-656.

14. Jacomy H, Fragoso G, Almazan G, Mushynski WE, Talbot PJ. (2006) Human coronavirus OC43 infection induces chronic encephalitis leading to disabilities in BALB/C mice. Virology. 349:335-346.

15. Sutton TC, Subbarao K. (2015) Development of animal models against emerging coronaviruses: from SARS to MERS coronavirus. Virology. 479-480:247-258. 